Research Article

\title{
Molecular Typing of Klebsiella pneumoniae Clinical Isolates by Enterobacterial Repetitive Intergenic Consensus Polymerase Chain Reaction
}

\author{
Parinaz Sedighi $\left(\mathbb{D},{ }^{1,2}\right.$ Omid Zarei $\left(\mathbb{D},{ }^{3}\right.$ Kiana Karimi ${ }^{D},{ }^{1,2}$ Mohammad Taheri $\left(\mathbb{D},{ }^{3}\right.$ \\ Pezhman Karami $\left(\mathbb{D},{ }^{3}\right.$ and Leili Shokoohizadeh $\left(\mathbb{D}^{3}\right.$ \\ ${ }^{1}$ Student Research Committee, Hamadan University of Medical Sciences, Hamadan, Iran \\ ${ }^{2}$ Universal Scientific Education and Research Network (USERN), Tehran, Iran \\ ${ }^{3}$ Department of Microbiology, Faculty of Medicine, Hamadan University of Medical Sciences, Hamadan, Iran
}

Correspondence should be addressed to Leili Shokoohizadeh; shokoohizadeh@yahoo.com

Received 6 September 2020; Revised 24 October 2020; Accepted 9 November 2020; Published 21 November 2020

Academic Editor: Simona Nardoni

Copyright (c) 2020 Parinaz Sedighi et al. This is an open access article distributed under the Creative Commons Attribution License, which permits unrestricted use, distribution, and reproduction in any medium, provided the original work is properly cited.

\begin{abstract}
Aim. Klebsiella pneumoniae is one of the most important causes of nosocomial infections, including pneumonia, sepsis, and urinary tract infection. Enterobacterial repetitive intergenic consensus polymerase chain reaction (ERIC-PCR) technique is a quick, reliable, and cost-effective method for molecular typing of Enterobacteriaceae family members. This study aimed to detect genetic relatedness among $K$. pneumoniae isolates from hospitals in Hamadan city, using ERIC-PCR technique. Materials and Methods. A total of $72 \mathrm{~K}$. pneumoniae isolates were collected from patients admitted to Besat and Sina hospitals. After detection and confirmation of $K$. pneumonia isolates by chemical and conventional microbiological methods, DNAs were extracted after 24 hours of incubation at $37^{\circ} \mathrm{C}$, using the boiling method. ERIC-PCR technique was carried out, and the ERIC patterns were analyzed by online data analysis service (inslico.ehu.es). ERIC profiles were compared using Dice method and clustered by UPGMA (unweighted pair group method with arithmetic mean) program. Also, the samples were evaluated by PCR method for the detection of aerobactin gene within their genome. Finding. The genetic relatedness among K. pneumoniae isolates was studied, and results established the genetic diversity of the clinical isolates by detecting 25 different ERIC types, including 14 common types and 11 unique types. Also, none of the isolates had aerobactin gene. Discussion. The results of this study showed high genetic diversity among K. pneumoniae strains, indicating the polyclonal distribution of $K$. pneumoniae isolates in Hamadan hospitals. This diversity causes problems for the treatment of infections due to the circulation of diverse K. pneumoniae clones, which possibly have different antimicrobial susceptibility patterns.
\end{abstract}

\section{Introduction}

Enterobacteriaceae family is a group of rod-shaped Gramnegative bacteria. Klebsiella pneumoniae, as the most important member of the Klebsiella genus, belongs to this family. K. pneumoniae is a part of human gastrointestinal normal flora and one of the most important organisms associated with opportunistic infections, especially among immunocompromised individuals. The pathogenesis of infection stands on various virulence factors such as polysaccharide capsule which protects bacteria against host defense, endotoxins, and iron-scavenging systems [1].

Nosocomial infections are developed after hospital admission besides the main health problem and are known as a global health issue. Multidrug-resistant pathogens usually are involved in such infections, and K. pneumoniae is one of 
the main culprits associated with pneumonia, urinary tract infection, wound infection, bacteremia, and septicemia [2-4].

One of the important points to prevent the spread of healthcare-associated infections and improve infection control is identifying the genetic relatedness among the pathogenic spices. Repetitive element sequence-based polymerase chain reaction (PCR) is a molecular method that investigates the repetitive nucleotide sequences within the bacterial genome and clusters the bacterial strains. Enterobacterial repetitive intergenic consensus (ERIC) is one of the repetitive elements in which the pattern and number are different in the bacterial genome. Enterobacterial repetitive intergenic consensus polymerase chain reaction (ERICPCR) technique is a quick, reliable, and cost-effective method for molecular typing of the Enterobacteriaceae family and distinguishes the genetic diversity among the strains $[4,5]$.

The aerobactin gene encodes the aerobactin siderophore which is one of the main iron-scavenging systems produced by hypervirulent $K$. pneumoniae. Hypervirulent strains can lead to life-threatening infections even in healthy individuals $[6,7]$.

This study aimed to detect genetic relatedness among $K$. pneumoniae isolates from hospitals in Hamadan city, using ERIC-PCR technique. Also, the presence of the aerobactin gene within their genomes was evaluated.

\section{Method}

2.1. Bacterial Isolation and Identification. A total number of $72 \mathrm{~K}$. pneumoniae isolates were gathered from all throat, urine, trachea, wound, blood, sputum, and abscess drainage samples entered to Besat and Sina Hospital laboratories from March to October 2019. Isolates were confirmed as $K$. pneumoniae by chemical and conventional microbiological methods at hospital laboratories. Also, antibiotic susceptibility testing was done by Kirby-Bauer disk diffusion method (Mast, UK) for amikacin, ceftazidime, ciprofloxacin, cotrimoxazole, imipenem, meropenem, levofloxacin, and colistin. The samples were saved in microtubes and frozen at $-70^{\circ} \mathrm{C}$ till all were gathered. Then, samples were cultured and incubated at $37^{\circ} \mathrm{C}$ for 24 hours. Samples' information including the sample type (urine, tracheal discharge, sputum, blood, wound, and abscess drainage), age and sex of patients, and type of admission (inpatient or outpatient) were extracted from laboratories' information systems.

2.2. ERIC-PCR. Genomic DNAs of K. pneumoniae were extracted using the boiling method. ERIC-PCR technique was carried out in a thermocycler (Bio-Rad, Inc. USA) using ERIC primer-forward: 5'-ATG TAA GCT CCT GGG GAT TCAC-3' and reverse: 5'-AAG TAA GTG ACT GGG GTG AGC G3' (Metabion, Germany). The PCR protocol is mentioned in Table 1 [8].

The PCR products were loaded on a one percent agarose gel (Sigma-Aldrich) at 70 volts for one hour, and the banding patterns were illuminated by ultraviolet radiation (Figure 1).
TABLE 1: ERIC-PCR protocol.

\begin{tabular}{lccc}
\hline PCR step & Temperature $\left({ }^{\circ} \mathrm{C}\right)$ & Time $(\mathrm{min})$ & Cycles \\
\hline Primary denaturation & 94 & 3 & 1 \\
Denaturation & 94 & 1 & 35 \\
Annealing & 48 & 1 & 35 \\
Extension & 72 & 2 & 35 \\
Final extention & 72 & 5 & 1 \\
\hline
\end{tabular}

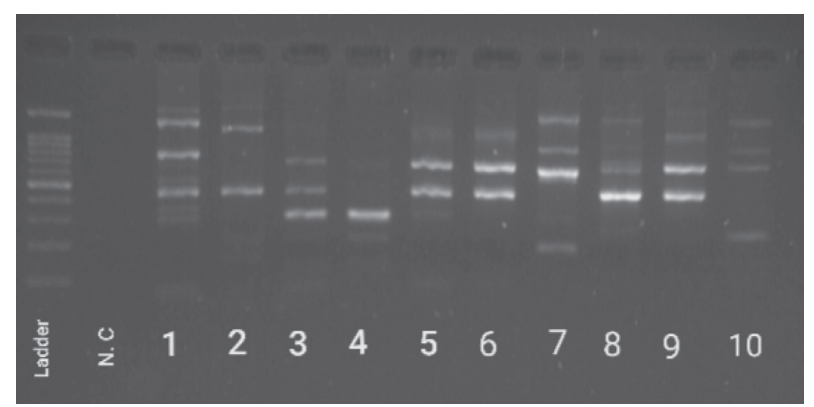

FIgURE 1: Agarose gel after electrophoresis and ultraviolet illumination. N. C: Negative control.

The ERIC patterns were analyzed by online data analysis service (insilico.ehu.es). ERIC profiles were compared using Dice method and clustered by UPGMA (unweighted pair group method with arithmetic mean) program. Isolates with a similar ERIC pattern or with one, two, or three different bands were considered as one ERIC type, and isolates with more than three inconsistent bands were fallen into different groups. The dendrogram was drawn according to the clusters. The relation between different ERIC types and hospitals in which samples were taken was evaluated by the Chi-square test using SPSS 22 software.

2.3. Detection of the Aerobactin Gene. The 72 samples were evaluated by PCR method for the detection of aerobactin gene within their genome. PCR was carried out in a thermocycler (Bio-Rad, Inc. USA) using the primer-forward: 5'-GCA TAG GCG GAT ACG AAC AT-3' and reverse: $5^{\prime}$ CAC AGG GCA ATT GCT TAC CT-3' (Metabion, Germany). The PCR protocol for detection of aerobactin gene is mentioned in Table 2 [9].

\section{Results}

A total number of 72 samples were entered into the study after isolates were confirmed as K. pneumoniae by Besat and Sina Hospital laboratories. 49 samples belonged to males. 6 samples belonged to outpatients. 47 samples belonged to inpatients, and type of admission was not determined for 19 samples. Among samples from inpatients, 40 samples were taken from patients in the intensive care unit (ICU). Also, there was one sample from patients admitted to each of hematology, infectious disease, orthopedics, general surgery, pediatrics and, burn wards. The ward was not determined for one of the inpatient samples. Of the total samples, 28, 15, 13, $5,5,1$, and 1 were extracted from the throat, urine, trachea, wound, blood, sputum, and abscess cultures, respectively 
TABLE 2: PCR protocol for detection of aerobactin gene.

\begin{tabular}{lccc}
\hline PCR step & Temperature $\left({ }^{\circ} \mathrm{C}\right)$ & Time $(\mathrm{min})$ & Cycles \\
\hline Primary denaturation & 95 & 5 & 1 \\
Denaturation & 95 & 1 & 40 \\
Annealing & 52 & 1 & 40 \\
Extension & 72 & 2 & 40 \\
Final extention & 72 & 7 & 1 \\
\hline
\end{tabular}

(Figure 2). According to antibiotic susceptibility testing, $86.5 \%, 84 \%, 80.6 \%, 72.5 \%, 72.3 \%, 67.3 \%$, and $66.1 \%$ of $K$. pneumoniae isolates were resistant to levofloxacin, ceftazidime, ciprofloxacin, cotrimoxazole, meropenem, amikacin, and imipenem, respectively. All of isolates were sensitive to colistin.

The genetic relatedness among $K$. pneumoniae isolates was studied, and results established 25 different ERIC types, including 14 common types and 11 unique types (Figure 3).

ERIC types A, B, C, G, L, and M belonged to Besat Hospital, while type $\mathrm{N}$ and most of type K samples belonged to Sina Hospital, and there was a significant difference between different ERIC groups in terms of the hospitals in which the samples were taken $(p$ value $<0.001)$. The largest ERIC type was type $\mathrm{L}$ including 10 isolates that all of them were from the intensive care unit (ICU) of Besat Hospital.

$72 \mathrm{~K}$. pneumoniae isolates were also accessed for the detection of aerobactin gene. Finally, none of the isolates had aerobactin gene.

\section{Discussion}

Gram-negative bacilli are one of the main causes of hospitalacquired infections. K. pneumoniae is one of the Gramnegative bacilli that belong to the Enterobacteriaceae family and the bacteria are associated with urinary tract infections, pneumonia, wound infections, and bloodstream infections $[2,4]$. K. pneumoniae is known as the third leading cause of hospital-acquired pneumonia in the United States, and also it is one the important causes of ventilator-associated pneumonia (VAP) in intensive care units (ICUs) $[10,11]$.

In our study, approximately $60 \%$ of isolates were related to the respiratory system, and about $20 \%$ of isolates were extracted from urine samples. In the same study by Parsaie Mehr et al. $70 \%$ of K. pneumoniae isolates were related to urine samples and $14 \%$ were extracted from tracheal samples [5]. In a prospective cohort study by Xercavins et al. to analyze the transmission dynamics of extended-spectrum $\beta$-lactamase-producing K. pneumoniae, among 60 isolates, about $47 \%$ were extracted from urine samples and only one was from respiratory tract sample [12]. The study of biofilm formation and antibiotic resistance of $K$. pneumoniae isolates from clinical samples by Nirwati et al. revealed that among 167 isolates, gathered from a tertiary care hospital, $51.5 \%$ were extracted from respiratory specimens [13]. We can conclude that respiratory and urinary tract infections are two important infections associated with $K$. pneumoniae.

Regarding the important role of $K$. pneumoniae in nosocomial infections, genotyping the clinical isolates is beneficial for the identification of infection sources and to

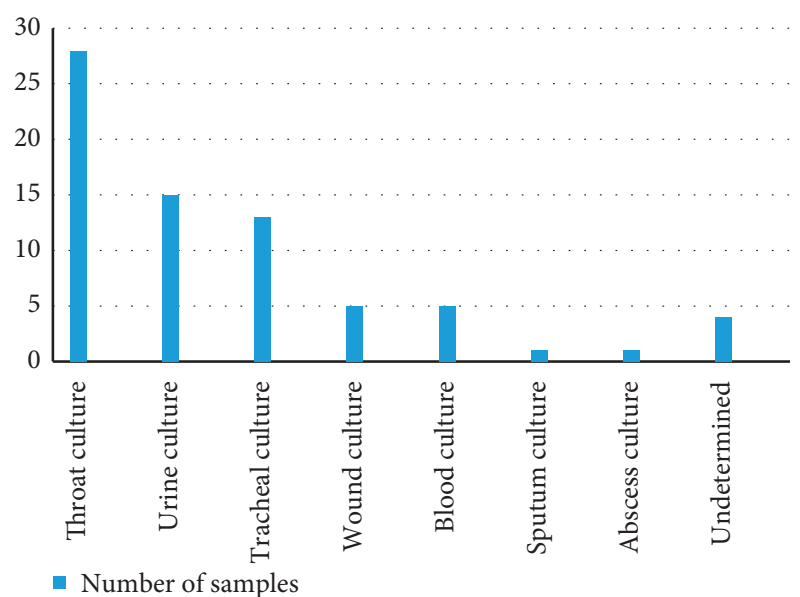

Figure 2: Number of different samples types.

prevent hospital-acquired infections [4]. Various molecular methods such as ribotyping, pulsed-field gel electrophoresis (PFGE), and multilocus sequence typing (MLST) can be implemented for genotyping bacterial spices. However, they are expensive and time-consuming. Repetitive element palindromic (REP) PCR method such as ERIC-PCR is another quick, reliable, and cost-effective technique for molecular typing of the Enterobacteriaceae family so that we applied this technique is our study. According to the results, 25 different ERIC types, including 14 common types and 11 unique types, were detected by ERIC-PCR which demonstrates the genetic diversity of the $K$. pneumoniae isolates from Hamadan hospitals. Samples of the largest ERIC group belonged to the ICU of a specific hospital, so this result expresses a dominant bacterial colon and possible nosocomial infection in this unit. According to our results, there was no significant relationship between ERIC groups and antibiotic susceptibility patterns. Studies in Iran and other countries have discussed genetic relatedness among $K$. pneumoniae isolates from different clinical settings. In the study by Moosavian et al. which was conducted on colistinresistant $K$. pneumoniae clinical isolates in the southwest of Iran, 23 ERIC types were identified among 26 spices [2]. The other study in Iran detected 32 different ERIC types among $35 \mathrm{~K}$. pneumoniae spices [5]. Also, Wasfi et al. evaluated genetic relatedness of multidrug-resistant $K$. pneumoniae isolates from Egyptian hospitals, and their results showed a diversity of the spices [14].

A study of virulence profiles of $54 \mathrm{~K}$. pneumoniae strains from various clinical specimens by Fertas-Aissani et al. reported the prevalence of aerobactin gene which is 3.7\% [15]. Also, another study by Remya et al. reported the prevalence of aerobactin gene to be $5.4 \%$ among 370 different clinical isolates [16]. However, aerobactin gene was detected in none of $K$. pneumoniae isolates in our study.

Our results in accordance with similar studies indicate genetic diversity of K. pneumoniae clinical isolates. Diverse colons may have different antibiotic resistance patterns and so can cause difficulties for the treatment of infections. We recommended future studies to evaluate genetic diversity among bacteria of the Enterobacteriaceae family and find 


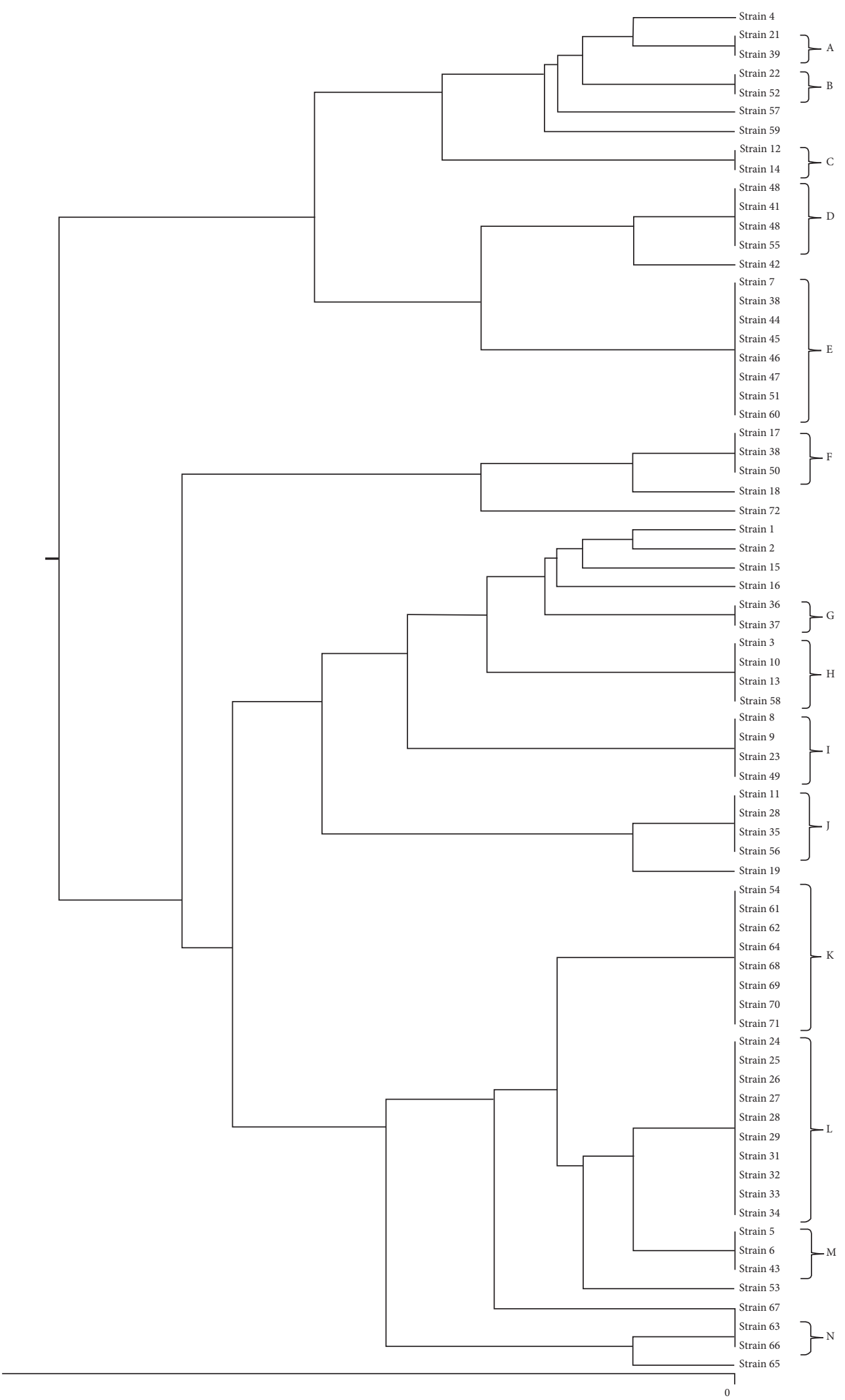

FIgURE 3: Dendogram of $\mathrm{k}$ pneumoniae isolates clustering based on ERIC patterns. 
possible relations between ERIC type and antibiotic resistance pattern. Also, we suggest further studies to evaluate more samples and the possible relations between expression of aerobactin gene and potential virulence of $K$. pneumoniae isolates.

\section{Data Availability}

Data of this manuscript are gathered from the research entitled "Molecular Typing of Klebsiella pneumoniae Isolates from Hamadan hospitals by Enterobacterial Repetitive Intergenic Consensus (ERIC)-PCR in 2018-2019.”

\section{Ethical Approval}

The study was approved by the ethics committee of Hamadan University of Medical Sciences (IR.UMSHA.REC.1397.682).

\section{Conflicts of Interest}

The authors declare that there are no conflicts of interest regarding the publication of this article.

\section{Acknowledgments}

The authors would like to thank staff of microbiology laboratory at Hamadan University of Medical Sciences. This research was supported by student research committee, Hamadan University of Medical Sciences (grant number: 9711096717).

\section{References}

[1] S. Zhang, G. Yang, Q. Ye, Q. Wu, J. Zhang, and Y. Huang, "Phenotypic and genotypic characterization of Klebsiella pneumoniae isolated from retail foods in China," Frontiers in Microbiology, vol. 9, no. 289, 2018.

[2] M. Moosavian and N. Emam, "The first report of emerging mobilized colistinresistance ( $\mathrm{mcr}$ ) genes and ERIC-PCR typing in Escherichia coli and Klebsiella pneumoniae clinical isolates in southwest Iran," Infection and Drug Resistance, vol. 12, pp. 1001-1010, 2019.

[3] H. Y. P. Phoon, H. Hussin, B. M. Hussain et al., "Distribution, genetic diversity and antimicrobial resistance of clinically important bacteria from the environment of a tertiary hospital in Malaysia," Journal of Global Antimicrobial Resistance, vol. 14, pp. 132-140, 2018.

[4] K. SeifiH. Kazemian et al., "Evaluation of biofilm formation among Klebsiella pneumoniae isolates and molecular characterization by ERIC-PCR," Jundishapur Journal of Microbiology, vol. 9, no. 1, 2016.

[5] V. Parsaie Mehr, L. Shokoohizadeh, M. Mirzaee, and M. Savari, "Molecular typing of Klebsiella pneumoniae isolates by enterobacterial repetitive intergenic Consensus (ERIC)-PCR," Infection Epidemiology and Microbiology, vol. 3, no. 4, pp. 112-116, 2017.

[6] T. A. Russo, R. Olson, U. MacDonald, J. Beanan, and B. A. Davidson, "Aerobactin, but not yersiniabactin, salmochelin, or enterobactin, enables the growth/survival of hypervirulent (hypermucoviscous) Klebsiella pneumoniae," Infection and Immunity, vol. 83, no. 8, pp. 3325-3333, 2015.
[7] T. A. Russo, R. Olson, U. MacDonald et al., "Aerobactin mediates virulence and accounts for increased siderophore production under iron-limiting conditions by hypervirulent (hypermucoviscous)Klebsiella pneumoniae ex vivo and in vivo," Infection and Immunity, vol. 82, no. 6, pp. 2356-2367, 2014.

[8] O. Zarei, L. Shokoohizadeh, H. Hossainpour, and M. Y. Alikhani, "Molecular analysis of Pseudomonas aeruginosa isolated from clinical, environmental and cockroach sources by ERIC-PCR," BMC Research Notes, vol. 11, no. 1, p. 668, 2018.

[9] W.-L. Yu, W.-C. Ko, K.-C. Cheng, C.-C. Lee, C.-C. Lai, and Y.-C. Chuang, "Comparison of prevalence of virulence factors for Klebsiella pneumoniae liver abscesses between isolates with capsular K1/K2 and non-K1/K2 serotypes," Diagnostic Microbiology and Infectious Disease, vol. 62, no. 1, pp. 1-6, 2008.

[10] R. M. Martin and M. A. Bachman, "Colonization, infection, and the accessory genome of Klebsiella pneumoniae," Frontiers in Cellular and Infection Microbiology, vol. 8, no. 4, 2018.

[11] S. S. Magill, J. R. Edwards, W. Bamberg et al., "Multistate point-prevalence survey of health care-associated infections," New England Journal of Medicine, vol. 370, no. 13, pp. 1198-1208, 2014.

[12] M. Xercavins, E. Jiménez, E. Padilla et al., "High clonal diversity of ESBL-producing Klebsiella pneumoniae isolates from clinical samples in a non-outbreak situation. A cohort study," Antimicrobial Resistance \& Infection Control, vol. 9, no. 1, p. $5,2020$.

[13] H. Nirwati, K. Sinanjung, F. Fahrunissa et al., "Biofilm formation and antibiotic resistance of Klebsiella pneumoniae isolated from clinical samples in a tertiary care hospital, Klaten, Indonesia," BMC Proceedings, vol. 13, no. 11, p. 20, 2019.

[14] R. Wasfi, W. F. Elkhatib, and H. M. Ashour, "Molecular typing and virulence analysis of multidrug resistant Klebsiella pneumoniae clinical isolates recovered from Egyptian hospitals," Scientific Reports, vol. 6, no. 1, p. 38929, 2016.

[15] R. El Fertas-Aissani, Y. Messai, S. Alouache, and R. Bakour, "Virulence profiles and antibiotic susceptibility patterns of Klebsiella pneumoniae strains isolated from different clinical specimens," Pathologie Biologie, vol. 61, no. 5, pp. 209-216, 2013.

[16] P. A. Remya, M. Shanthi, and U. Sekar, "Characterisation of virulence genes associated with pathogenicity in Klebsiella pneumoniae," Indian Journal of Medical Microbiology, vol. 37, no. 2, pp. 210-218, 2019. 